\title{
Ouabain Induces Apoptotic Cell Death in Type I Spiral Ganglion Neurons, but not Type II Neurons
}

\author{
H. Lang, ${ }^{1}$ B. A. Schulte, ${ }^{1,2}$ And R. A. Schmiedt ${ }^{2}$ \\ ${ }^{1}$ Department of Pathology and Laboratory Medicine, Medical University of South Carolina, Charleston, SC 29425, USA \\ ${ }^{2}$ Department of Otolaryngology—Head and Neck Surgery, Medical University of South Carolina, Charleston, SC 29425, USA
}

Received: 18 March 2004; Accepted: 16 November 2004; Online publication: 2 February 2005

\begin{abstract}
Application of ouabain to the intact round-window (RW) membrane of the gerbil cochlea induces apoptosis in most spiral ganglion neurons (SGNs), leaving a few neurons intact (Schmiedt et al. 2002). Here, physiological measures and immunostaining were used to examine the process of SGN degeneration at $3,6,12$, and $24 \mathrm{~h}, 4$ days, and 1 and 5 months after ouabain treatment. The few remaining neurons surviving up to 5 months after ouabain treatment were immunoreactive for peripherin, a type II neuron marker. Peripherin-positive cell counts indicate that about $7 \%$ of the SGNs in the gerbil cochlea are type II neurons, and these neurons survive intact after ouabain treatment. Ouabain exposure had little effect on the outer hair cell and lateral wall systems, even after a 5 month loss of auditory-nerve function. The cellular locations of cytochrome $c$, poly (ADP-ribose) polymerase (PARP), and activated caspase 3 were examined in control and ouabain-treated cochleas. A redistribution of cytochrome $c$ in peripherinnegative (type I) neurons was observed at $3 \mathrm{~h}$ after ouabain exposure. Degraded PARP and activated caspase 3 were also detected in peripherin-negative SGNs at 6 and $24 \mathrm{~h}$ after treatment, respectively. These results suggest that the redistribution of cytochrome $c$ is an early event during apoptosis in type I SGNs and that activation of PARP and caspase 3 are associated with apoptosis in these cells. Calcineurin and NF-кB are two important signaling pathways that may
\end{abstract}

Correspondence to: H. Lang - Department of Pathology and Laboratory Medicine $\cdot$ Medical University of South Carolina $\cdot 165$ Ashley Avenue - PO Box 250908 Charleston, SC 29425, USA. Telephone: +1-843-7922711; fax: +1-843-7920368; email: langh@ musc.edu modulate cell survival in the central nervous system. Here, we found that calcineurin and NF- $\mathrm{KB}$ selectively labeled type II neurons. It is speculated that the high levels of calcineurin and NF-kB in type II SGNs, as compared with type I SGNs, may play protective roles in enhancing the survival of type II neurons exposed to ouabain.

Keywords: spiral ganglion neuron, apoptosis, cytochrome $c$, caspase 3 , PARP, calcineurin

\section{INTRODUCTION}

Apoptosis is known to be involved in neuronal death during normal development, in many neurodegenerative disorders and with exposure to neurotoxic agents. An ultrastructural study by Dodson found that type I neurons in the spiral ganglion were lost mainly through the process of apoptosis after intracochlear injection of gentamicin (Dodson 1997). Recently, we have shown that application of ouabain to the round-window (RW) membrane of the cochlea results in a rapid loss of spiral ganglion neurons (SGNs; Schmiedt et al. 2002). Physiological measurements using this model of auditory-nerve neuropathy demonstrated a complete absence of the compound action potential (CAP) response across all frequencies. Conversely, endocochlear potentials (EPs) and distortion product otoacoustic emissions (DPOAEs) were largely unaffected by ouabain treatment. Apoptosis was evident in SGNs 12 to $24 \mathrm{~h}$ after short-term ouabain exposure as indicated both by TdT-biotin nick end labeling (TUNEL) and direct observation of nuclear morphology. Because the survival of SGNs is a critical issue in age-related hearing loss and in the 
success of cochlear implants, a better understanding of the mechanism of SGN death is required to formulate ways to prevent auditory neuropathy.

Mitochondria play critical roles in neuronal energy metabolism and in the regulation of cellular $\mathrm{Ca}^{2+}$ homeostasis. Cytochrome $c$ is an essential component of the mitochrondrial respiratory chain and normally is compartmentalized in the mitochrondrial intermembrane space. In response to various cell-death stimuli, changes in mitochondria membrane permeability and abnormal levels of intracellular $\mathrm{Ca}^{2+}$ result in the release of cytochrome $c$ into the cytosol and nucleus. Cytochrome $c$ and procaspase 9, together with apoptosis-activating factor-1, form a multiprotein complex that activates caspase 9 , which then cleaves procaspase 3 into active caspase 3. Active caspase 3, together with other activated downstream caspases, proteolytically degrades a variety of proteins, inducing the morphological alterations characteristic of apoptosis (for review, see Fiskum 2000; Fiskum et al. 2003). PARP also participates in apoptotic cell death as reported recently (Yu et al. 2003). PARP may induce apoptosis through the formation of large amounts of poly ADP-ribose (PAR) and the depletion of $\beta$-nicotinamide adenine dinucleotide $\left(\mathrm{NAD}^{+}\right)$. Together, these events may directly trigger apoptosis by a cascade of events, such as mitochondrial depolarization, release of mitochondrial apoptosisinitiating factor, and cytochrome $c$, and subsequent caspase activation (Ha and Snyder 2000; Chiarugi and Moskowitz 2002; Yu et al. 2003). Here, immunohistochemistry was used to determine the cellular expression and location of PARP, cytochrome $c$, and activated caspase 3 in SGNs of control and ouabaintreated gerbils.

There are two subpopulations of neurons in the spiral ganglion of the mammalian cochleas. Large type I neurons with peripheral processes synapsing on inner hair cells represent approximately $90-95 \%$ of the afferent auditory neurons and are myelinated. The remaining small type II neurons innervate the outer hair cells and are unmyelinated (Spoendlin 1969; Kiang et al. 1982, 1984). Type II neurons are characterized by an accumulation of intermediate filament proteins. Peripherin is one member of family of intermediate filament proteins and is considered a reliable marker for type II neurons (Berglund and Ryugo 1986; Hafidi 1998). Our previous study demonstrated that a small number of neurons with small nuclei survive after acute ouabain treatment (Schmiedt et al. 2002). Immunostaining revealed that these surviving neurons were peripherin-positive. Calcineurin and NF- $\mathrm{KB}$ are two important signaling molecules that can respond to extracellular stress or receptor-activated signal transduction and modulate cell survival (Morioka et al. 1992, 1997, 1999; Mattson et al. 2000; Lilienbaum and Israël 2003). Our hypothesis is that the presence or absence of certain neuron-specific proteins (e.g., calcineurin or NF-кB) in type I and type II neurons determines their fate (death or survival) after exposure to certain toxic agents. Here, the cellular expression and distribution of calcineurin and NF- $\mathrm{BB}$ in type I and type II neurons were evaluated in normal and ouabaintreated cochleas.

\section{MATERIALS AND METHODS}

\section{Animal and surgical procedures}

Mongolian gerbils of both genders with healthy external ears were used for this study. The animals were born and raised in a low-noise environment where the median sound level was $40 \mathrm{~dB}$ SPL. Ages ranged from 4 to 8 months. The animal facilities have full AAALAC accreditation, and all experimental procedures were approved by the local IACUC and met NIH guidelines for animal care and use.

Animals were anesthetized with sodium pentobarbital $(50 \mathrm{mg} / \mathrm{kg})$ and given atropine $(0.2 \mathrm{mg} / \mathrm{kg})$ to reduce the respiratory secretions. Body temperature was maintained between 36 and $38^{\circ} \mathrm{C}$ using a heating pad. Sterile procedures were used to apply ouabain to the round window (RW) wherein about 20-40 $\mu \mathrm{l}$ of a $1 \mathrm{mM}$ ouabain solution in normal saline was placed in RW niche for $30 \mathrm{~min}$. The ouabain was removed by a small piece of filter paper before closing the surface of the bulla. The surface of the bulla was then fully closed with dental cement, and incisions were closed with sutures. Animals were allowed to recover for 3, 6, 12, and $24 \mathrm{~h}, 4$ days, and 1 and 5 months. Postsurgical discomfort was treated with buprenorphine if necessary. The untreated left ear was used as a control.

\section{Physiological procedures}

The procedures for recording the CAP response, DPOAEs, and EP were similar to those previously described (Hellstrom and Schmiedt 1996; Schmiedt et al. 2002; Lang et al. 2003). Physiological data were obtained from both ears. The animal was anesthetized with sodium pentobarbital as described above and fitted to a head holder in a sound- and vibrationisolated booth. The pinna and surrounding tissues were removed and the bulla opened widely. The CAP electrode was placed on the bony rim of the RW niche, and the acoustic assembly, comprising a probetube microphone (B\&K 4134, Bruel and Kjaer, Norcross, GA) and driver (Beyer DT-48, Beyerdy- 
namic, Farmingdale, NY), was sealed to the bony ear canal with closed-cell foam. The tone pips were generated in the frequency domain by Tucker Davis Technologies (Alachua, FL) equipment and software. CAP thresholds were obtained visually with an oscilloscope on line at half-octave frequencies from 0.5 to $20 \mathrm{kHz}$ with tone pips of $1.8 \mathrm{~ms}$ total duration with $\cos ^{2}$ rise/fall times of $0.55 \mathrm{~ms}$. EPs were recorded in cochlear turns 1 (T1) and 2 (T2), corresponding to best frequencies of about 16 and $2.2 \mathrm{kHz}$ according to the single-fiber map of Müller (1996). The EP was measured with a micropipet filled with $0.2 \mathrm{M} \mathrm{KCl}$ yielding an impedance of approximately 20-30 M $\Omega$. The output of the micropipet was led to an electrometer (World Precision Instruments FD 223) for direct recording of the potential. EP was defined as the voltage difference between scala media and a pool of isotonic saline on the neck muscles. The micropipet was introduced into scala media via 50-70 $\mu \mathrm{m}$ holes drilled through the otic capsule in $\mathrm{T} 1$ and $\mathrm{T} 2$.

\section{Morphological procedures}

After the physiological recording, anesthetized animals were perfused via cardiac catheter with $10 \mathrm{ml}$ of normal saline containing $0.1 \%$ sodium nitrite followed by $50 \mathrm{ml}$ of fixative solution of $10 \%$ formalin and $0.5 \%$ zinc dichromate in $0.9 \%$ saline with the $\mathrm{pH}$ adjusted to 5.0 just prior to use. The inner ears were then dissected, immersed in fixative for $45 \mathrm{~min}$, decalcified with EDTA, dehydrated, embedded in paraffin, and sectioned at $6 \mu \mathrm{m}$ thickness.

The tissue sections were deparaffinized, dehydrated, and immersed in a $3 \%$ hydrogen peroxide solution for $15 \mathrm{~min}$ at room temperature to inactivate endogenous peroxidase. Tissues were antigen retrieval treated by boiling for $10 \mathrm{~min}$ in $10 \mathrm{mM}$ citrate buffer, $\mathrm{pH}$ 6.0, followed by cooling for $20 \mathrm{~min}$. After immersion in blocking solution for $20 \mathrm{~min}$, the sections were incubated overnight with a primary antibody diluted in PBS at $4^{\circ} \mathrm{C}$. The primary antibodies used in this study are listed in Table 1. Secondary antibodies were biotinylated, and binding was detected with HRP techniques visualized with DAB or by labeling with fluorescein-conjugated avidin D. Nuclei were counterstained with hematoxylin for sections visualized with $\mathrm{DAB}$ or with bis-benzimide for sections visualized with fluorescein.

Dual immunolabeling was used for colocalization of cytochrome $c$ and peripherin, calcineurin and pheripherin, and calcineurin and NF- $\mathrm{B}$ p65. The double-labeling procedure followed the basic protocol of VECTAgram Labeling of Multiple Antigens (Vector, Burlingame, CA). Sections were incubated overnight with one primary antibody diluted in PBS at $4^{\circ} \mathrm{C}$. The secondary antibody was biotinylated and binding was detected with fluorescein-conjugated avidin D (1:100) (Vector). The Avidin/Biotin Blocking kit (SP-2001, Vector) was used before the procedure for the secondary antigen. The procedure for detection of the secondary antigen with double labeling was the same as for the first antigen, except TexasRed-conjugated avidin D (1:100) (Vector) was used for visualization. Negative controls, where the primary antibody was omitted, were routinely evaluated to assay the specificity of all the secondary antibodies. No specific labeling was detected in any of these control experiments. The sections were mounted with VECTASHIELD mounting medium for fluorescence (Vector).

Sections were examined on a Zeiss Axioplan research microscope (Carl Zeiss Inc., Jena, Germany) equipped with a $100 \mathrm{~W}$ mercury light source and a $25 \times$ plan-neoflur na 1.3 objective. Some sections stained for cytochrome $c$ were examined on a Zeiss LSM5 Pascal confocal microscope (Carl Zeiss Inc.)

\section{TABLE 1}

Sources, dilutions, and characterization of antibodies

\begin{tabular}{|c|c|c|c|c|c|}
\hline Antibodies & Provider & Host & Dilution & Specificity & $\begin{array}{c}\text { Antigen-retrieval } \\
\text { treatment }\end{array}$ \\
\hline Cytochrome $C$ & Chemicon $^{\mathrm{a}}$ & Mouse & $1: 100$ & 15 kDa cytochrome $c^{b}$ & Yes \\
\hline PARP (N-20) & Santa Cruz $^{\mathrm{C}}$ & Goat & $1: 100$ & 112 kDa PARP & Yes \\
\hline Active caspase 3 & R\&D system ${ }^{\mathrm{d}}$ & Rabbit & $1: 500$ & p17 subunit caspase $3^{b}$ & Yes \\
\hline Peripherin & Chemicon $^{\mathrm{a}}$ & Rabbit & $1: 100$ & $\sim 57$ kDa peripherin ${ }^{\mathrm{b}}$ & No \\
\hline Peripherin & Chemicon $^{\mathrm{a}}$ & Mouse & $1: 100$ & $\sim 57$ kDa peripherin ${ }^{\mathrm{b}}$ & No \\
\hline PP2B-A (H-209) (calcineurin) & Santa Cruz ${ }^{\mathrm{C}}$ & Rabbit & $1: 100$ & A subunit calcineurin ${ }^{b}$ & No \\
\hline
\end{tabular}

${ }^{a}$ Chemicon International Inc. (Temecula, CA).

${ }^{\mathrm{b}}$ The information on the specificity of antibody has been provided by manufacture.

'Santa Cruz Biotechnology Inc. (Santa Cruz, CA).

${ }^{\mathrm{d}} \mathrm{R} \& \mathrm{D}$ Systems Inc. (Minneapolis, MN). 
with an $\mathrm{AR}$ and HeNe laser. The captured images were processed using Image Pro Plus software (Media Cybernetics, MD), Zeiss LSM Image Browser Version 3.2.0.70 (Carl Zeiss Inc.) and Adobe Photoshop 7.0.

\section{RESULTS}

Physiology

Figure 1 shows the CAP thresholds and EP values at different recovery times after ouabain exposure. Control data are the means of all untreated ears. After $3 \mathrm{~h}$ of recovery, there were no CAP responses at 16 and $20 \mathrm{kHz}$, whereas those between 0.5 and 2
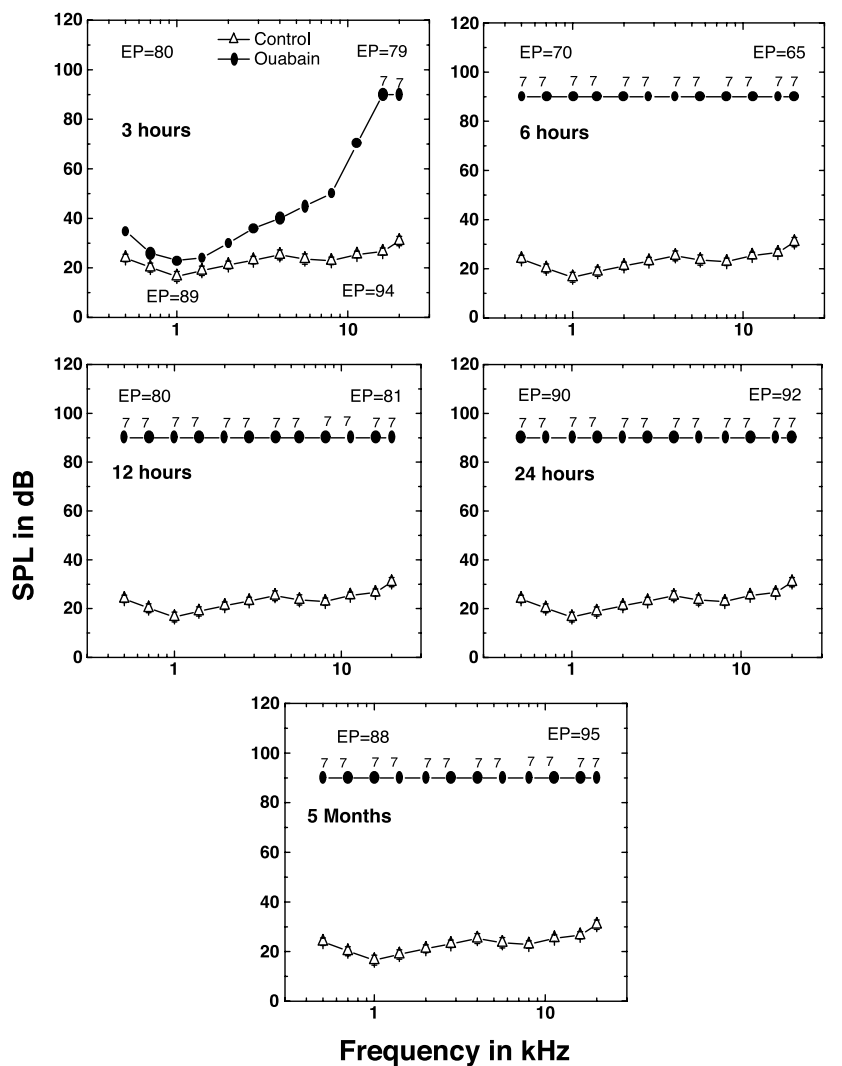

FIG. 1. CAP and EP recorded in control and ouabain-treated ears. Individual data from $3,6,21$, and $24 \mathrm{~h}$ and 5 months of recovery after treatment are plotted as filled circles. Mean control data (open triangles) were obtained from the untreated left ears. Error bars represent standard errors of the mean. The EPs were measured in the basal ( $\mathrm{T} 1 \mathrm{at} 16 \mathrm{kHz}$ ) and middle ( $\mathrm{T} 2$ at $2.2 \mathrm{kHz}$ ) turns. There is a sharp demarcation in CAP thresholds after $3 \mathrm{~h}$ of recovery, changing from nearly normal thresholds at frequencies of 0.5 and $1 \mathrm{kHz}$ to no response at the frequencies of 16 and $20 \mathrm{kHz}$. EP after $3 \mathrm{~h}$ was only slightly decreased from control values. Panels depicting data after 6 , 12 , and $24 \mathrm{~h}$ and 5 months of recovery demonstrate that the CAP response is absent across all frequencies in the treated ears. The EP values show some decrease after $6 \mathrm{~h}$, but rebound to normal values after $24 \mathrm{~h}$ of recovery and remain normal up to 5 months after exposure to ouabain.
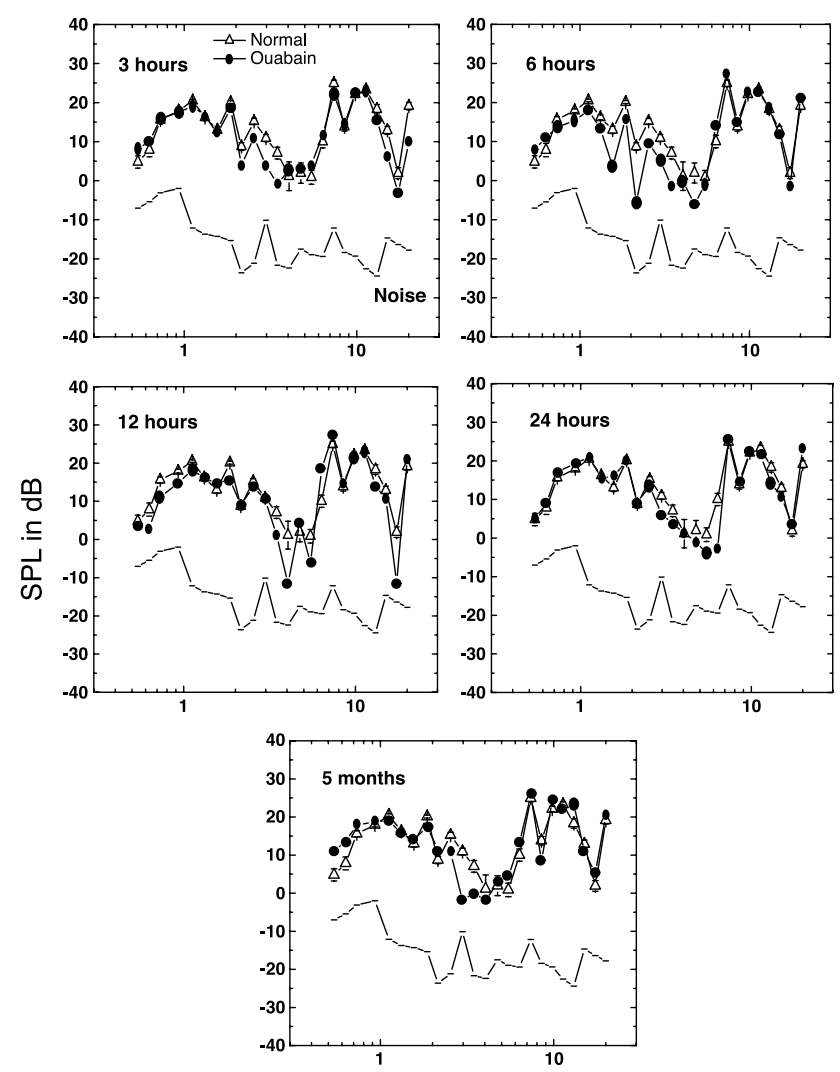

Frequency of $\mathrm{f}_{2}$ in $\mathrm{kHz}$

FIG. 2. DPOAEs obtained with constant level $(\mathrm{L} 1=\mathrm{L} 2=50 \mathrm{~dB}$ SPL) primaries from control (open triangles) and ouabain-treated (filled circles) ears. Error bars represent standard errors of the mean for control values. Ears are the same as those shown in Figure 1. DPOAEs remain similar to control values for all recovery times after ouabain exposure.

$\mathrm{kHz}$ were nearly normal in threshold. After $3 \mathrm{~h}$, EPs were less than the control means by about $15 \mathrm{mV}$ at $\mathrm{T} 1$ and $9 \mathrm{mV}$ at T2. After $6 \mathrm{~h}$, EPs at both T1 and T2 declined about $19 \mathrm{mV}$. The CAP response was completely absent across all frequencies in the treated ears after 6,12 , and $24 \mathrm{~h}, 4$ days, and 1 and 5 months. The EP showed the largest decline after $6 \mathrm{~h}$, but recovered to normal values after $24 \mathrm{~h}$ and remained near normal at 5 month postouabain treatment. Thus, an acute ouabain exposure to RW induced a complete and irreversible loss of auditory-nerve function with only a small transient loss of EP.

"DPOAE grams" obtained with constant-level primaries from the same ears shown in Figure 1 are plotted in Figure 2. The primary level used in this study were $50 \mathrm{~dB}$ SPL. DPOAEs obtained with these low-level primaries are sensitive indicators of outer hair cell function. DPOAEs remained similar to control values for all recovery times up to 5 months after ouabain treatment. It is clear that the ouabain exposure had little effect on the outer hair 
cell system, even after a 5 month loss of auditorynerve function.

\section{Translocation of cytochrome $c$ and apoptosis}

Morphological hallmarks of apoptosis, such as shrunken cytoplasm and condensed pyknotic nuclei, are readily observable in hematoxylin- and eosin-stained or hematoxylin-counterstained sections (Kerr et al. 1995; Lang et al. 2000). The left panel of Figure 3 illustrates the progressive apoptosis in SGNs induced by ouabain exposure. Three hours after treatment, neuronal cell bodies showed a slight shrinkage with no observable nuclear pathological profiles (Fig. 3B). Pyknotic nuclei were present after $12 \mathrm{~h}$, other neurons still maintained normal nuclear morphology (Fig. 3C). At $24 \mathrm{~h}$, most neurons showed marked apoptotic changes, although a few with small nuclei still showed normal morphological profiles (Figs. 3D, H).

Cytochrome $c$ is normally compartmentalized in the space between the outer and inner membranes of mitochondria (Reed 1997). Mitochrondria are uniformly distributed in the soma of SGNs (Adamo and Daigneault 1973; Ylikoski et al. 1978). Normal SGNs show a weak but uniform cytoplasmic staining for cytochrome $c$ (Figs. 3E, 4A). Three hours after treatment, many SGNs showed intense immunostaining for cytochrome $c$ (Figs. 3F, 4B). After 12 h, cytoplasmic diffuse staining for cytochrome $c$ is present in some neurons (Figs. 3G, 4C). At the same time, some of the SGNs showed morphological changes indicative of apoptosis (Fig. 3C). At $24 \mathrm{~h}$ postouabain exposure, cytochrome $c$ was not detected in neurons with late-phase apoptosis. A few small neurons with a normal appearing morphology showed uniform staining as in the controls (Fig. 3H). Five-month postouabain exposure, there were similar staining patterns as those seen at $24 \mathrm{~h}$ (data not shown). The redistribution of cytochrome $c$ staining after ouabain treatment indicates that the translocation of cytochrome $c$ is an early event during ouabain-induced apoptosis in SGNs.

In other regions of the inner ear including stria vascularis, sensory hair cells, and type II and IV fibrocytes of the spiral ligament, cytochrome $c$ labeling revealed patterns consistent with mitochrondial distribution and did not change with ouabain treatment (data not shown).

\section{Type II neurons surviving after ouabain treatment}

Our previous results have shown that application of ouabain onto the RW of cochlea induces apoptosis in most SGNs, leaving a few neurons intact (Schmiedt et al. 2002). Peripherin, an intermediate filament protein, is considered a reliable marker for type II
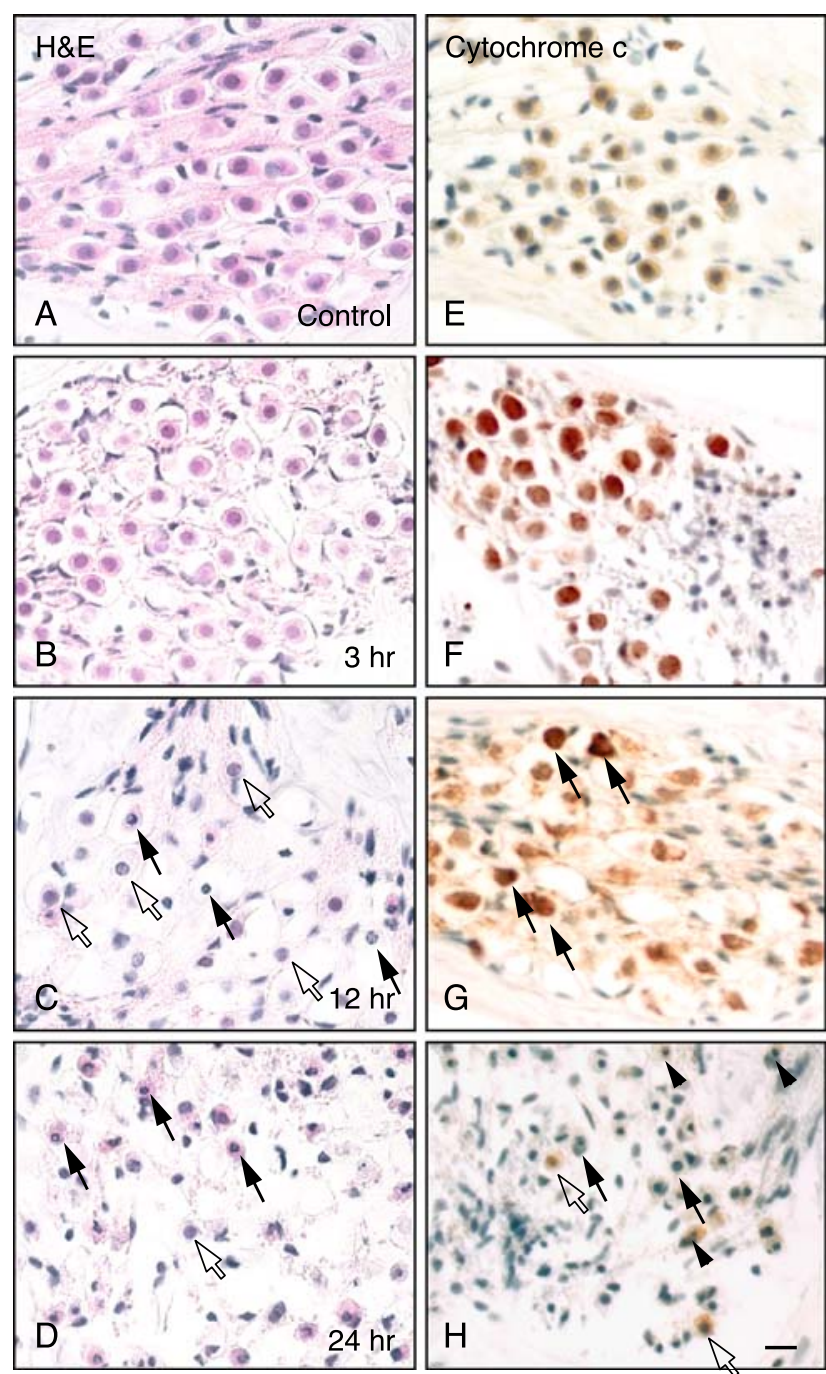

FIG. 3. Cytochrome $c$ is associated with apoptosis in SGNs. Ears are the same those shown in Figures 1 and 2. All photos are taken from midmodiolar sections of the basal turn. Right panels show the expression of cytochrome $c$ in control and ouabain-treated ears; nuclei are counterstained with hematoxylin. Left panels show H\&E staining in the same location of the same cochlea. (A) H\&E of control ear shows normal profiles of ganglion cell bodies. (B) Slight shrinkage is seen in the neuronal cell bodies at $3 \mathrm{~h}$ posttreatment. (C) At $12 \mathrm{~h}$ posttreatment, classic signs of apoptosis (filled arrows) are present in some of the ganglion-cell nuclei. Open arrows indicate normal profiles of ganglion neurons. (D) After $24 \mathrm{~h}$, most neuronal nuclei show apoptotic profiles (filled arrows), whereas a few normal neurons with small nuclei are found scattered among dying neurons (open arrows). (E) In control tissue, staining for cytochrome $c$ is uniform throughout the cytoplasm of SGNs and is clearly absent in the nuclear space. (F) Three hours after ouabain exposure, there is an intense labeling of cytochrome $c$ throughout the cytoplasm and nuclear space. (G) Twelve hours after, some SGNs show diffuse staining for cytochrome $c$ (filled arrows). (H) Twenty-four hours after, cytochrome $c$ is not detectable in most neuronal profiles exhibiting condensed nuclear morphology (filled arrows); however, a few neurons with condensed nuclei do show weak cytochrome $c$ staining (filled arrowheads). A few neurons with normal small nuclei also show cytoplasmic staining for cytochrome $c$ (opened arrows). Scale bar $=10 \mu \mathrm{m}$. 

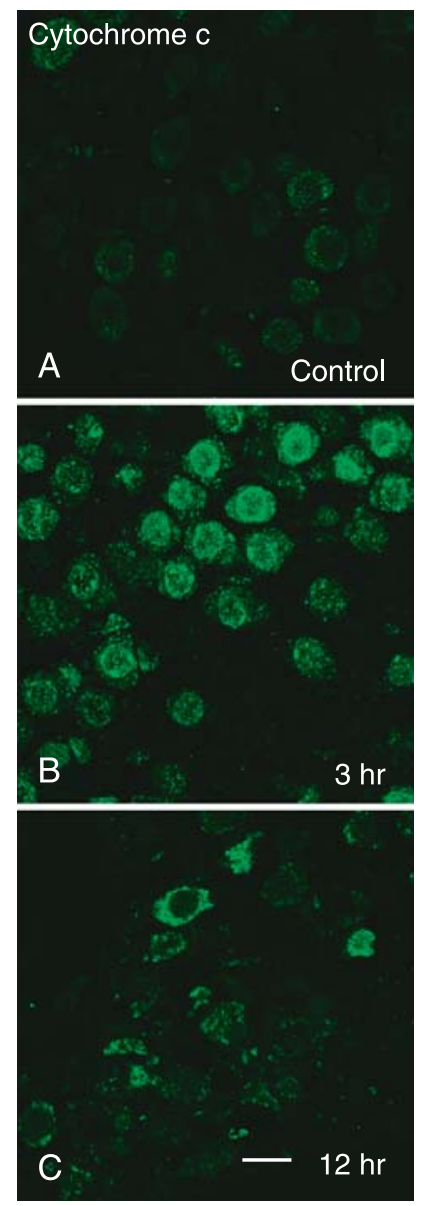

FIG. 4. Confocal images of cytochrome $c$ staining as fluorescein (green) in the basal turns of control and ouabain-treated ears. (A) In normal ears, there is a weak staining in the cytoplasm of most SGNs. (B) Three hours after ouabain treatment, strong staining for cytochrome $C$ is seen, both in the cytoplasm and nuclei of most SGNs. (C) At $12 \mathrm{~h}$ postexposure, strong diffuse staining remains in the cytoplasm of a few neurons. Scale bar $=10 \mu \mathrm{m}$.

SGNs (Hafidi 1998). A small number of peripherinpositive neurons were found in control and in 3, 12, and $24 \mathrm{~h}, 4$ day, and 1 and 5 month postouabaintreated cochleas (Figs. 5, 7, 8, Table 2). To determine whether some type II neurons were lost after ouabain treatment, the peripherin-positive cells were counted in the midmodiolar sections of treated and control cochleas (Table 2). No significant differences were noted in their positive cell counts nor in their physiological data; thus, an overall average was obtained to compare against sections from the untreated control ears. The mean numbers of peripherin-positive cells per section in T2, T1 (upper), and T1 (lower) in ouabain-treated cochleas were $3.6 \pm 0.6,2.0 \pm 0.4$ and $2.0 \pm 0.4$, respectively, whereas the numbers in the respective control cochleas were $2.3 \pm 0.7,2.0 \pm 0.7$ and $2.5 \pm 0.6$, respectively. Although sample sizes were too small for rigorous statistical comparison of the number of type II neurons in control and treated cochleas, the data suggest no obvious loss of type II neurons after ouabain treatment. The results also indicate that approximately $7 \%$ of the ganglion cell population in the normal gerbil ear are type II neurons and these neurons survive after ouabain exposure.

\section{Differential distribution of cytochrome $c$ in type I and type II neurons}

Dual immunostaining demonstrated that the cytochrome $c$ redistribution induced by ouabain did not occur in peripherin-positive SGNs (Fig. 5). The type II neurons presented a consistent pattern of weak and uniform cytochrome $c$ staining in control and all ouabain-treated cochleas. These data suggest that

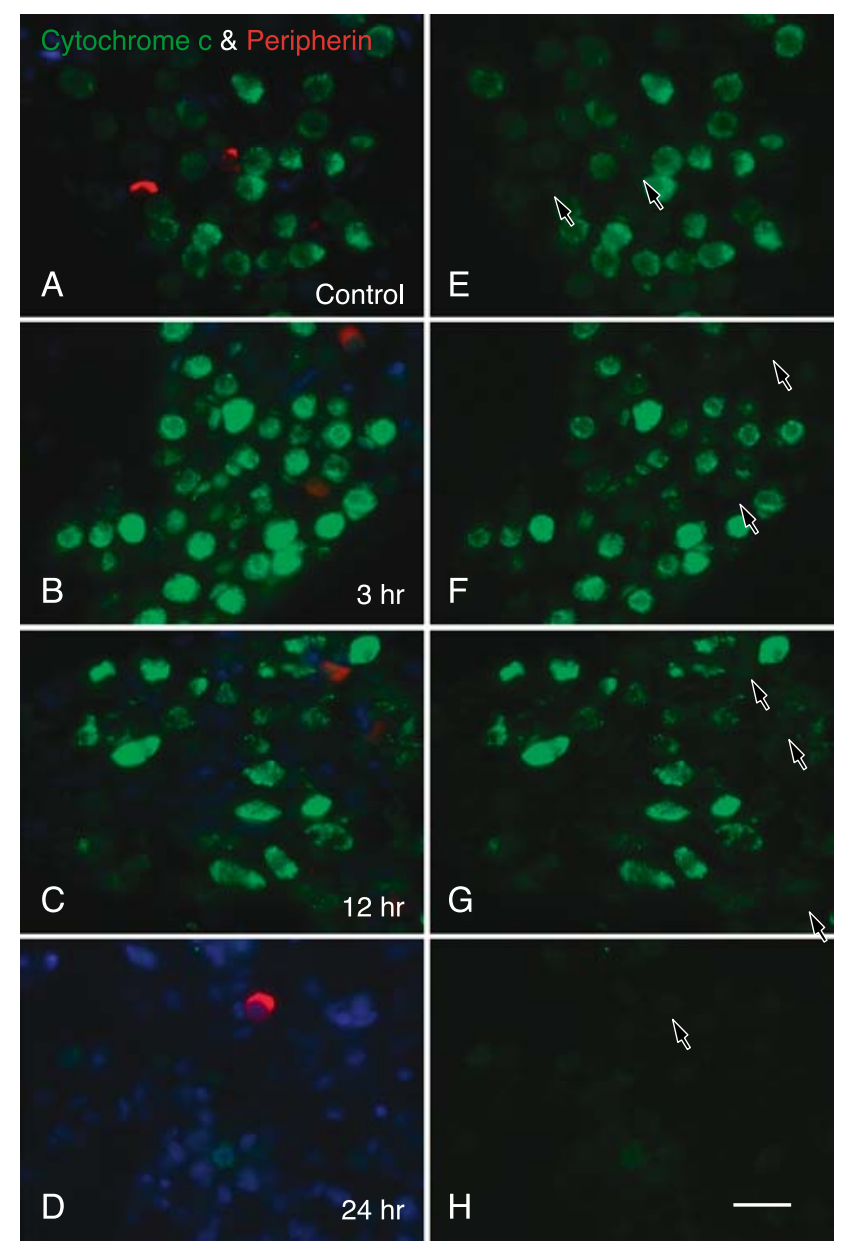

FIG. 5. Differential distribution of cytochrome $c$ in type I and type II SGNs. Peripherin, a marker for type II neurons, was detected using Texas Red (red). Cytochrome $c$ was detected using fluorescein (green). Nuclei were counterstained with bis-benzimide (blue). All photos were taken from midmodiolar sections of the basal turns. (A-D) A strong signal for cytochrome $c$ is present only in neurons which failed to stain for peripherin. (E-H) Images are the same as (A-D), but with only fluorescein filter. The staining intensity and cellular locations of cytochrome $c$ are not affected with ouabain treatment in peripherin-positive neurons (open arrows). Scale bar = $15 \mu \mathrm{m}$. 
TABLE 2

\begin{tabular}{|c|c|c|c|c|c|c|}
\hline \multicolumn{7}{|c|}{ Peripherin-positive cell counts in midmodiolar sections of gerbil cochleas after ouabian exposure } \\
\hline \multirow[b]{2}{*}{ Recovery time } & \multirow[b]{2}{*}{ Section } & \multirow[b]{2}{*}{ Turn } & \multicolumn{2}{|c|}{ Right ear (treated) } & \multicolumn{2}{|c|}{ Left ear (control) } \\
\hline & & & $\begin{array}{l}\text { Positive cells } \\
\text { (total SG cells) }\end{array}$ & Percentage & $\begin{array}{l}\text { Positive cells } \\
\text { (total SG cells) }\end{array}$ & Percentage \\
\hline 4 days & 1 & T2 & $3(3)$ & 100 & $0(24)$ & 0 \\
\hline 4 days & 1 & T1 (upper) & $1(1)$ & 100 & $1(29)$ & 3.4 \\
\hline 4 days & 1 & T1 (hook) & $2(2)$ & 100 & $1(24)$ & 4.2 \\
\hline 4 days & 2 & $\mathrm{~T} 2$ & $6(6)$ & 100 & $6(49)$ & 12.2 \\
\hline 4 days & 2 & T1 (upper) & $2(2)$ & 100 & $2(29)$ & 6.9 \\
\hline 4 days & 2 & T1 (hook) & $1(1)$ & 100 & $2(29)$ & 6.9 \\
\hline 4 days & 3 & $\mathrm{~T} 2$ & $6(6)$ & 100 & $3(32)$ & 9.3 \\
\hline 4 days & 3 & T1 (upper) & $1(1)$ & 100 & $2(28)$ & 7.1 \\
\hline 4 days & 3 & T1 (hook) & $0(0)$ & 100 & $2(31)$ & 6.5 \\
\hline 4 days & 4 & $\mathrm{~T} 2$ & $4(4)$ & 100 & $2(28)$ & 7.1 \\
\hline 4 days & 4 & T1 (upper) & $3(3)$ & 100 & $0(29)$ & 0 \\
\hline 4 days & 4 & T1 (hook) & $3(3)$ & 100 & $4(30)$ & 13.3 \\
\hline 4 days & 5 & $\mathrm{~T} 2$ & $2(2)$ & 100 & $2(27)$ & 7.4 \\
\hline 4 days & 5 & T1 (upper) & $1(1)$ & 100 & $1(29)$ & 3.4 \\
\hline 4 days & 5 & T1 (hook) & $2(2)$ & 100 & $0(24)$ & 0 \\
\hline 1 month & 1 & $\mathrm{~T} 2$ & $4(4)$ & 100 & $1(26)$ & 3.8 \\
\hline 1 month & 1 & T1 (upper) & $3(3)$ & 100 & $7(58)$ & 12.1 \\
\hline 1 month & 1 & T1 (hook) & $4(4)$ & 100 & $2(27)$ & 7.4 \\
\hline 1 month & 2 & $\mathrm{~T} 2$ & $3(3)$ & 100 & $2(28)$ & 7.1 \\
\hline 1 month & 2 & T1 (upper) & $4(4)$ & 100 & $1(42)$ & 2.4 \\
\hline 1 month & 2 & T1 (hook) & $3(3)$ & 100 & $5(31)$ & 16.1 \\
\hline 5 months & 1 & $\mathrm{~T} 2$ & $0(0)$ & $\mathrm{N} / \mathrm{A}$ & $5(41)$ & 12.2 \\
\hline 5 months & 1 & T1 (upper) & $1(1)$ & 100 & $2(49)$ & 4.1 \\
\hline 5 months & 1 & T1 (hook) & $2(2)$ & 100 & $2(20)$ & 10 \\
\hline 5 months & 2 & $\mathrm{~T} 2$ & $4(4)$ & 100 & $0(25)$ & 0 \\
\hline 5 months & 2 & T1 (upper) & $2(2)$ & 100 & $3(28)$ & 11 \\
\hline 5 months & 2 & $\mathrm{~T} 1$ (hook) & $1(1)$ & 100 & $5(23)$ & 22 \\
\hline
\end{tabular}

acute application of ouabain to the RW induces a release of cytochrome $c$ from mitochrodria in type I SGNs, but not in type II neurons.

\section{PARP and active caspase 3 in type I neurons}

PARP is highly expressed in the nucleus, with about one molecule per 1,000 DNA base pairs (Skaper 2003; Chiarugi and Moskowitz 2002). During apoptosis, the PARP can cleave into a stable $85 \mathrm{kDa}$ active fragment containing the carboxyl terminal and a 25 kDa fragment (Lazebnik et al. 1994). The PARP antibody used here reacts specifically with the 112 $\mathrm{kDa}$ intact form of PARP and does not detect the $85 \mathrm{kDa}$ active fragment of the cleavage. In control ears, immunoreactive staining for PARP was uniformly localized to the nuclei and absent in the cytoplasm of SGNs (Figs. 6A, 7A). At $6 \mathrm{~h}$ postouabain treatment, staining intensity for PARP was markedly reduced in many neurons, none of which were peripherinpositive (Figs. 6B, 7B). A few neurons showed normal levels of PARP labeling, and one of them was a peripherin-positive neuron (Figs. 6B, 7B). At 12 and $24 \mathrm{~h}$ postouabain treatment when most neurons are apoptotic or already dead, staining for PARP was absent in most neurons, but not in the peripherinpositive cells (data not shown). These data indicate that the degradation of PARP is an early event in the apoptosis of type I neurons.

Caspases are synthesized as inactive proenzymes that are processed to an active form in cells undergoing apoptosis. The caspase 3 antibody used here preferentially recognizes the p17 fragment of active caspase 3. In control ears, no immunostaining of caspase 3 was present in the spiral ganglion (Figs. 6C, 7C). At $24 \mathrm{~h}$ postouabain treatment, staining for caspase 3 was localized to the cytoplasm of SGNs, all of which were peripherin-negative (Figs. 6D, 7D). We did not find caspase-3-positive neurons in controls or at 3,6 , and $12 \mathrm{~h}$ postouabain treatment (data not shown). There were very few neurons with caspase 3, although cytochrome $c$ redistribution was commonly seen in SGNs after ouabain treatment. Thus, the timing of caspase 3 activation and the cytochrome $c$ redistribution is different.

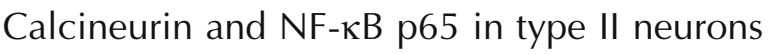

Calcineurin is a calmodulin-binding protein widely distributed in various tissues, but most abundant in 
the central nervous system (Nakazawa et al. 2001; Erin et al. 2003). The antibody used here was directed against calcineurin A (PP2B-A), a subunit of the $60 \mathrm{kDa}$ catalytic component. A low percentage of SGNs were intensely labeled with calcineurin in the normal gerbil cochlea (Fig. 8A). All calcineurinpositive neurons were also strongly immunopositive for peripherin (Figs. 8B, C) demonstrating that calcineurin is another reliable marker for type II neurons. All type II neurons maintained stable and showed strong calcineurin labeling at 3, 6, 12, and $24 \mathrm{~h}$ (data not shown) and 5 months after-ouabain exposure (Figs. 8D-F).

$\mathrm{NF}-\kappa \mathrm{B}$ is a homo- or heterodimer protein belonging to the NF-кB/Rel family and may consist of five subunits as identified in mammalian cells: RelA or P65, RelB, c-rel, p50, and p52. The p50/p65 complex is the most prominent and is considered to be the prototype of the NF-кB factors (Verma et al. 1995; Lilienbaum and Israël 2003). At equilibrium, the inactive form of $\mathrm{NF}-\kappa \mathrm{B}$ is located in the cytoplasm and is bound to inhibitory $\mathrm{I} \kappa \mathrm{B}$ molecules. Certain stimuli induce $\mathrm{I} \kappa \mathrm{B}$ to dissociate from the complex nucleus, and the activated $\mathrm{NF}-\kappa \mathrm{B}$ is translocated to the nucleus (Mattson et al. 2000). The antibody used here was against the p65 subunit of NF- $\kappa$ B. Strong immunostaining for $\mathrm{NF}-\kappa \mathrm{B}$ was selectively present in

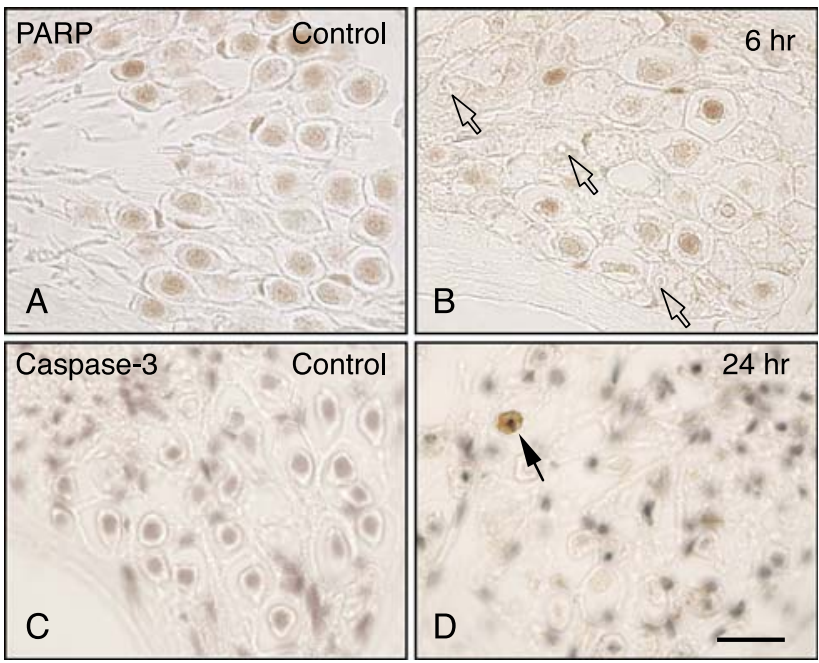

FIG. 6. Expression of intact PARP and activated caspase 3 in control and ouabain-treated ears. All photos were taken from midmodiolar sections of the basal turns. (A) In control ears, there is uniform weak to moderate staining in the nuclei of most SGNs. (B) Six hours after treatment, PARP immunoreactivity is weak or absent in many neurons (open arrows), whereas a few neurons still show normal levels of PARP staining. (C) No binding of antibody to activated caspase 3 was detected in control ears. (D) Twenty-four hours after ouabain treatment, staining for activated caspase 3 was seen in the cytoplasm of a ganglion cell (filled arrow) with a condensed nucleus. Nuclei were counterstained with hematoxylin in (C) and (D). Scale bar $=20 \mu \mathrm{m}$.
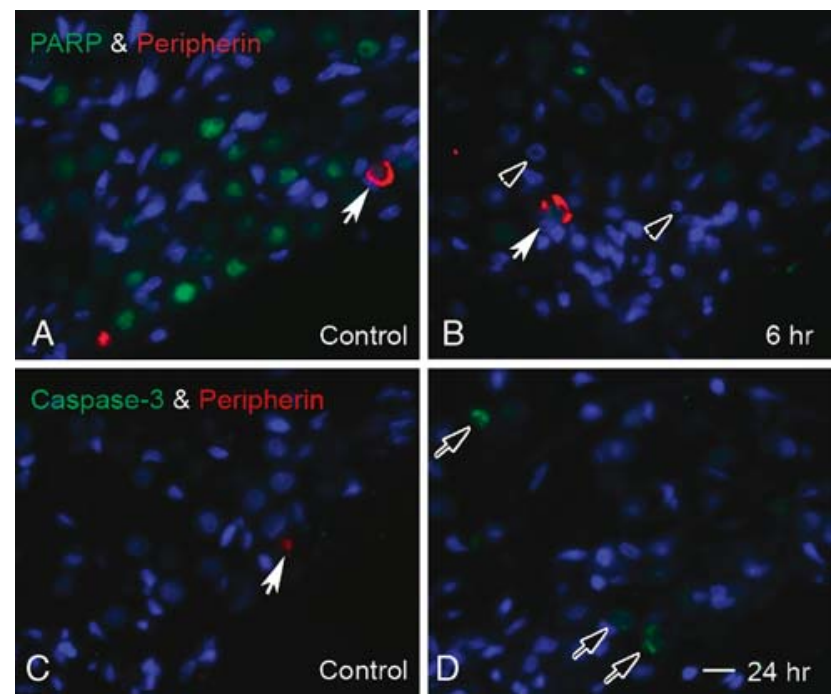

FIG. 7. Double labeling for PARP and activated caspase 3 with peripherin, respectively. PARP and activated caspase 3 were detected using fluorescein (green), whereas peripherin was using Texas Red (red). Nuclei were counterstained with bis-benzimide (blue). All photos were taken from midmodiolar sections of the basal turns. (A) In control ears, there is uniform weak to moderate staining in the nuclei of most SGNs including a type II neuron (filled arrow) for PARP. (B) Six hours after treatment, PARP immunoreactivity is weak or absent in most neurons, open arrowheads indicate neurons with pyknotic nuclei (arrowheads). A peripherin-positive neuron is seen with PARP staining (filled arrow). (C) No staining of activated caspase 3 was detected in neurons of control ears. A filled arrow indicates peripherin-positive cytoplasm in a type II neuron. (D) Twenty-four hours after ouabain treatment, staining for activated caspase 3 was seen in the cytoplasm of some peripherin-negative neurons (open arrows). Scale bar $=10 \mu \mathrm{m}$.

the cytoplasm of type II SGNs of all control and treatment ears (Fig. 9). Other regions of gerbil cochlea included the stria vascularis, fibrocytes of the spiral ligament beneath the stria vascularis, fibrocytes in suprastrial area, and limbus were also

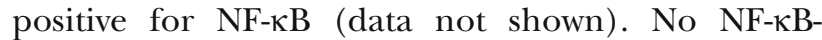
immunostaining was detected in the nucleus of control or ouabain-treated SGNs.

\section{DISCUSSION}

Degeneration of SGNs is a common pathologic feature correlated with permanent hearing loss. Such degeneration has been shown to occur in humans and animal models as the result of aging and cochlear injuries due to noise, ototoxic drugs, or genetically determined hair cell loss (Kiang et al. 1976; Spoendlin 1975, 1984; Keithley and Feldman 1979; Leak and Hradek 1988; Zimmerman et al. 1995; Dodson and Mohuiddin 2000; White et al. 2000). The majority of studies suggest that degeneration of the auditory nerve is a slow process that continues over months or 

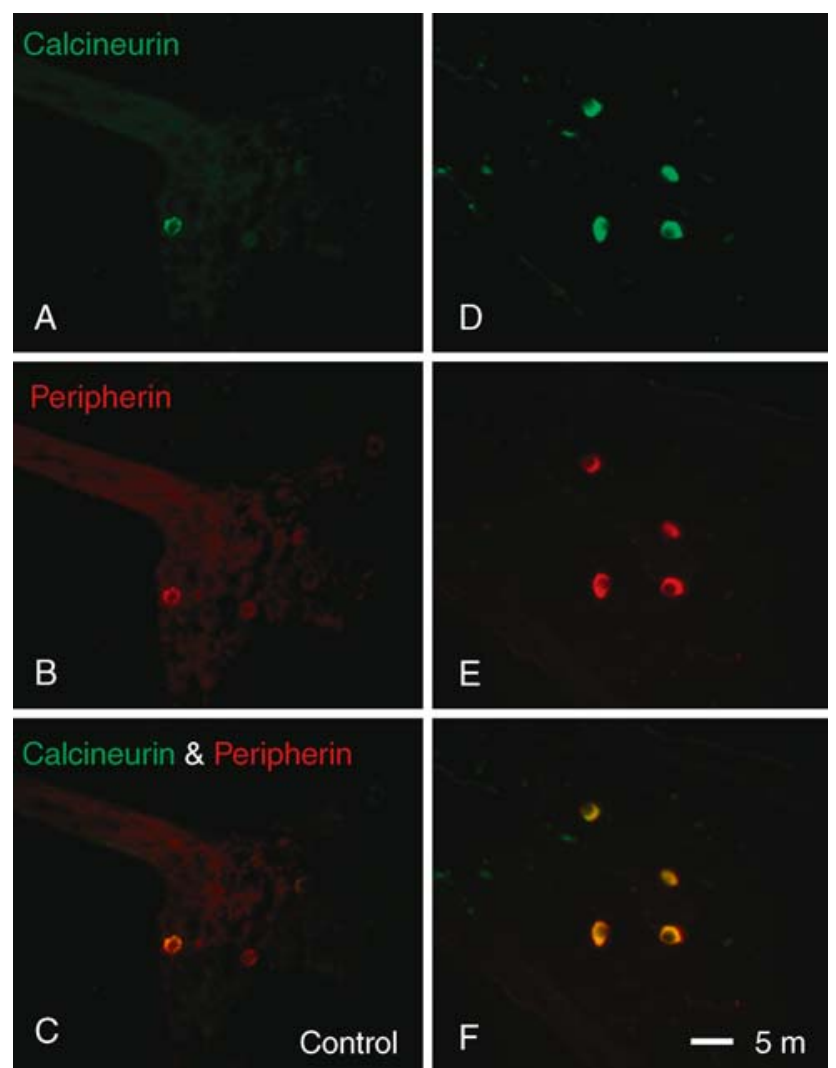

FIG. 8. Double labeling for peripherin and calcineurin in SGNs. Calcineurin was detected using fluorescein (green) and peripherin was detected using Texas Red (red). Left panels: control ear. All photos were taken from midmodiolar sections of the basal turns. (A) Only three neurons in this section are calcineurin-positive. (B) Same neurons shown in (A) are peripherin-positive. (C) Double exposure of the two antibodies (yellow). Right panel: ouabain-treated ear after 5 months of recovery. (D) All remaining neurons are calcineurinpositive. (E) Same neurons shown in (F) are peripherin-positive. (G) Double exposure of the two antibodies (yellow). Scale bar $=15 \mu \mathrm{m}$.

years, making it problematic to study the cellular mechanisms of the neuronal death. In contrast, acute application of oubain to the RW results in the death of most SGNs within 12-24 h and provides a reliable model to observe the process of SGN death in vivo. Using this model, we have demonstrated that ouabain-induced neuropathy in the gerbil cochlea is associated with the release of cytochrome $c$ and involves the cleavage of PARP and activation of caspase 3 in type I SGNs.
Many genes and signaling pathways are involved in apoptosis in various cell types. In neurons, the mitochrondrial mechanism of cell death has received particular attention. Ouabain is a cardiac glycoside that specifically binds to $\mathrm{Na}$, K-ATPase and blocks its activity. In vitro studies have shown that ouabaininduced apoptosis of cortical neurons is accompanied by intracellular increases of $\mathrm{Ca}^{+}$and $\mathrm{Na}^{+}$and by decreases in $\mathrm{K}^{+}$and cytochrome $c$ release (Xiao et al. 2002). Here, we used an immunohistochemical approach to examine the consequences of mitochrondrial cytochrome $c$ release in ouabain-induced apoptosis of gerbil SGNs. Three hours after ouabain treatment, most neurons showed an intense staining for cytochrome $c$ throughout the cytoplasm and nuclei; after $12 \mathrm{~h}$, there was diffuse staining in some neurons. By $24 \mathrm{~h}$ after treatment, most neurons showed signs of apoptosis, whereas the staining for cytochrome $c$ was weak or undetectable. Similar immunostaining patterns for cytochrome $c$ have been reported in cortical neurons as a consequence of transient focal ischemia, in embryonic lens fiber cells during differentiation, and in cochlear hair cells after noise and ototoxic drug damage (Nakagawa and Yamane 1999; Fujimura et al. 2000; Cheng et al. 2002; Nicotera et al. 2003).

PARP degradation and caspase 3 activation are two important processes related to mitochrondrial cytochrome $c$-mediated apoptosis. Degradation of intact PARP was detected in SGNs $6 \mathrm{~h}$ after exposure to ouabain. Subsequent to PARP cleavage and cytochrome $c$ redistribution, caspase 3 activation was present in apoptotic SGNs $24 \mathrm{~h}$ after ouabain exposure. These data indicate that PARP cleavage and cytochrome $c$ redistribution are early events in the apoptosis of SGNs (starting about 3-6 h after ouabain treatment).

Immunostaining for peripherin confirmed that the neurons surviving for up to 5 months after ouabain treatment are type II cells. The peripherin-positive cell counts in the midmodiolar sections of cochleas before and after treatment suggest that either none or very few of type II neurons are lost after ouabain exposure. Dual labeling of cytochrome $c$, PARP, and caspase 3 with peripherin demonstrate clearly that redistribution of cytochrome $c$, degradation of PARP, and activation of caspase 3 occurred only in type I and not in
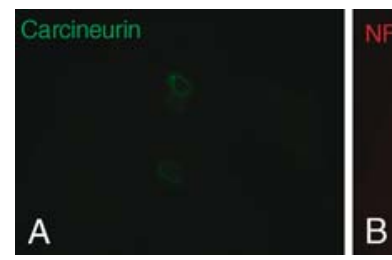

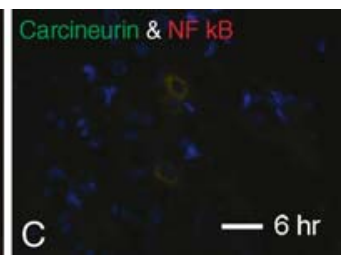

FIG. 9. Double labeling with calcineurin and NF- $\mathrm{kB}$ in SGNs $6 \mathrm{~h}$ after ouabain treatment. Calcineurin was detected using fluorescein (green) and NF- $\mathrm{\kappa B}$ was detected using Texas Red (red). Nuclei were counterstained with bis-benzimide (blue). All photos were taken from midmodiolar sections of the basal turns. (A) Three neurons are calcineurin-positive. (B) Same neurons shown in (A) are NF-KB-positive. Double exposure of the two antibodies (yellow). Scale bar $=15 \mu \mathrm{m}$. 
type II neurons. This result provides direct and indirect evidence that type II neurons are not affected by ouabain treatment. Our data also indicate that type II neurons account for about $7 \%$ of the SGNs in the gerbil cochlea.

Type II neurons have been shown to have some selective survival advantage over the type I neurons in cat after ototoxic lesions of the organ of Corti (Spoendlin 1975; Lim 1976; Bichler et al. 1983; Leak and Hradek 1988), noise trauma (Spoendlin 1975; Lim 1976), or transection of the cochlear nerve (Spoendlin 1975). Some of these studies also reported the presence of a new type of neuron (type III) after various insults. These rarely observed neurons were thought to be more like type I neurons based on their ultrastructural features including lack of a thick myelin sheath, a darker perikaryon than nucleus, a smooth round nucleus with an electron-dense nucleolus, a cytoplasm with relatively few neurofilaments, and an abundance of organelles (Spoendlin 1981; Leak and Hradek 1988). Type III neurons are quite rare in the normal cochlea and often have been considered to be demyelinated type I neurons in cochleas with hair cell loss. In the absence of ultrastructural studies, we are unable to eliminate the possibility that some surviving neurons were type III neurons. However, if type III neurons survive in the ouabain-treated gerbil ear, our data would suggest that they are more likely to derive from type II than type I SGNs, given their positive immunostaining for peripherin.

Calcineurin belongs to the family of $\mathrm{Ca}^{2+} /$ calmodulin-dependent protein phosphatases and is highly expressed in neurons in the hippocampus, striatum, substantia nigra, neocortex, and cerebellum (Wallace et al. 1980; Goto et al. 1986; Kincaid et al. 1987). Neurons subjected to variety of injuries, including ischemia, trauma, and ouabain exposure, often suffer from a large influx of $\mathrm{Ca}^{2+}$. Calcineurin may act to buffer $\mathrm{Ca}^{2+}$ and decrease intracellular free $\mathrm{Ca}^{2+}$ loads (Morioka et al. 1999). Previous studies have suggested that calcineurin may be involved in delayed neuronal death in the rat hippocampus after transient forebrain ischemia (Morioka et al. 1992, 1997; Yamasaki et al. 1992). Here, the relatively high levels of calcineurin in type II as compared with type I SGNs suggest that it may play a critical role in the survival of the type II neurons after exposure to ouabain.

In addition to possibly enhancing neuronal survival after ouabain exposure, our results show that calcineurin is a reliable marker for distinguishing type II from type I SGNs and may provide insight as into their function. Calcineurin is involved in longterm potentiation and depression of synaptic transmission and regulation of synaptic activity and receptor function in the central nervous system (Bliss and Collingridge 1993; Lisaman 1989; Mulkey et al. 1994). In the sensory neurons of Caenorhabditis elegans, calcineurin negatively regulates multiple aspects of sensory signaling including the thermosensory and olfactory responses (Kuhara et al. 2002).

Like calcineurin, immunoreactive NF-kB was detected at high levels in the cytoplasm of type II neurons. However, we failed to find any nuclear staining for NF-kB in control or treated ears, suggesting that it is not activated by ouabain exposure. Further studies using more sensitive techniques, such as gel-shift or supershift assays, for nuclear protein extracts may be needed to detect the activated form of NF- $\mathrm{B}$ (Yu et al. 1999).

In summary, our data demonstrate that: (1) the type II SGN population survives relatively intact after ouabain treatment; (2) the type II SGNs comprise about $7 \%$ of the SGNs in the gerbil; (3) the release of cytochrome $c$ is an early event during apoptosis in type I, ut not in type II SGNs; (4) degradation of intact PARP and activation of caspase 3 are both involved in cytochrome $c$-mediated apoptosis in type I SGNs; (5) the type II neurons can survive at least 5 months after ouabain exposure; (6) calcineurin is a reliable marker for type II gerbil SGNs; and (7) the high levels of calcineurin and NF- $\mathrm{KB}$ in type II SGNs may be associated with their survival after exposure to ouabain.

\section{ACKNOWLEDGMENTS}

This work was supported by grants NIH/NIA AG14748 (R.A.S) and NIH/NIDCD DC00713 (B.A.S.). We thank Nancy Smythe and Boo Schmiedt for their technical assistance.

\section{REFERENCES}

Adamo NJ, Daigneault EA. Ultrastructure features of neurons and nerve fibres in the spiral ganglia of cats. J. Neurocytol. 2:91-103, 1973

Berglund AM, Ryugo DK. A monoclonal antibody labels type II neurons of the spiral ganglion. Brain Res. 383:327-332, 1986.

Bichler E, Spoendin H, Raucheager H. Degeneration of cochlear neurons after amikacin intoxication in the rat. Arch. Otorhinolaryngol. 237:201-208, 1983.

Bliss TV, Collingride GL. A synaptic model of memory: long-term potential in the hippocampus. Nature 361:31-39, 1993.

Cheng AG, Cunningham LL, Rubel EW. Translocation of cytochrome $c$ in hair cells of gentamicin-treated avain basilar papilla. Assoc. Res. Otolaryngol. Abstr. 25:134, 2002.

Chingugi A, Moskowitz MA. PARP-1: a perpetrator of apoptotic cell death? Science 297:200-201, 2002.

Dodson HC. Loss and survival of spiral ganglion neurons in the guinea pig after intracochlear perfusion with aminoglycosides. J. Neurocytol. 26:541-556, 1997.

Dodson HC, Mohuiddin A. Response of spiral ganglion neurons to 
cochlear hair cell destruction in the guinea pig. J. Neurocytol. 29:525-537, 2000.

Erin N, Bronson SK, Billingsley ML. Calcium-dependent interaction of calcineurin with Bcl-2 in neuronal tissue. Neuroscience 117:541-555, 2003

Fiskum G. Mitochondrial participation in ischemic and traumatic neural cell death. J. Neurotrauma 17:843-855, 2000.

Fiskum G, Starkov A, Polster BM, Chinopoulos C. Mitochondrial mechanisms of neural cell death and neuroprotective interventions in Parkinson's disease. Ann. N. Y. Acad. Sci. 991:111-119, 2003.

Fujimura M, Morita-Fujimura Y, Noshita N, Sugawara T, Kawase M, Chan PH. The cytosolic antioxidant copper/zinc-superoxide dismutase prevents the early release of mitochondria cytochrome $c$ in ischemic brain after transient focal cerebral ischemia in mice. J. Neurosci. 20(8):2817-2824, 2000.

Goto S, Matsukado Y, Mihara Y, Inoue N, Miyamoto E. The distribution of calcineurin in rat brain by light and electron microscopic immunohistochemistry and enzyme-immunoassay. Brain Res. 397:161-172, 1986.

Ha HC, SNYDER SH. Poly (ADP-ribose) polymerase-1 in the nervous system (review). Neurobiol. Dis. 7:225-239, 2000.

HAFIDI A. Peripherin-like immunoreactivity in type II spiral ganglion cell body and projections. Brain Res. 805:181-190, 1998.

Hellstrom LI, Schmiedt RA. Measures of tuning and suppression in single-fiber and whole-nerve response in young and quiet-aged gerbils. J. Assoc. Soc. Am. 100:3275-3285, 1996.

Keithley EM, Feldman ML. Spiral ganglion cell counts in an agegraded series of rat cochleas. J. Comp. Neurol. 188:429-442, 1979.

Kerr JFR, Gobe GC, Winterford CM, Harmon BV. Anatomical methods in cell death. In: Schwartz LM, Osborne BA (eds) Methods in Cell Biology. Academic Press, San Diego, pp P1-P27, 1995.

Kiang NYS, Liberman MC, Levine RA. Auditory-nerve activity in cats exposed to ototoxic drugs and high-intensity sound. Ann. Otol. Rhinol. Laryngol. 85:752-768, 1976.

Kiang NYS, Rho JM, Northrop CC, Liberman MC, Ryugo DK. Hair cell innervation by spiral ganglion cells in adult cat. Science 217:129-177, 1982.

Kiang NYS, Liberman MC, Gage JS, Northrop CC, Dodds LW, Oliver ME. In: Bolis L, Deynes RD, Maddrel SHP (eds) Comparative Physiology of Sensory Systems. Cambridge University Press, New York, pp 143-161, 1984.

Kincaid RL, Balaban CD, Billingsley ML. Differential location of calmodulin-dependent enzymes in rat brain: evidence for selective expression of cyclic nucleotide phosphodiesterase in specific neurons. Proc. Natl. Acad. Sci. USA 84:1118-1122, 1987.

Kuhara A, InAda H, Katsura I, Mori I. Negative regulation and gain control of sensory neurons by the $C$. elegans calcineurin TAX- 6 . Neuron 33(5):751-763, 2002.

Lang H, Bever MM, Fekete DM. Cell proliferation and cell death in the developing chick inner ear: spatial and temporal patterns. J. Comp. Neurol. 417:205-220, 2000.

Lang H, Schulte BA, Schmiedt RA. Effects of chronic furosemide treatment and age on cell division in the adult gerbil inner ear. J. Assoc. Res. Otolaryngol. 4:164-175, 2003.

Lazebnik YA, Kaufmann SH, Desnoyers S, Poirier GG, Earnshaw WC. Cleavage of poly (ADP-ribose) polymerase by a proteinase with properties like ICE. Nature 371:346-347, 1994.

Leak PA, Hradek GT. Cochlear pathology of long term neomycin induced deafness in cats. Hear. Res. 33:11-34, 1988.

Lilienbaum A, IsRaËL A. From calcium to NF-kB signaling pathways in neurons. Mol. Cell. Biol. 23(8):2680-2698, 2003.

Liм DJ. Ultrastructural cochlear changes following acoustic hyperstimulation and ototoxicity. Ann. Otol. Rhinol. Laryngol. 85 (6 Pt 1):740-751, 1976.

Lisaman J. A mechanism of Hebb and anti-Hebb processes un- derlying learning and memory. Proc. Natl. Acad. Sci. USA 86: 9574-9578, 1989.

Mattson MP, Culmsee C, Yu Z, Camandola S. Roles of nuclear factor $\kappa \mathrm{B}$ in neuronal survival and plasticity. J. Neurochem. 74:443456, 2000.

Morioka M, Fukunaga K, Yasugawa S, Nagahiro S, Ushio Y, Мгуното E. Regional and temporal alterations in $\mathrm{Ca}^{2+}$, calmodulin-dependent proteins kinase II and calcineurin in the hippocampus of rat brain after transient forebrain ischemia. J. Neurochem. 58:1798-1809, 1992.

Morioka M, Nagahiro S, Fukunaga K, Miyamoto E, Ushio Y. Calcineurin in the adult rat hippocampus: different distribution in CA1 and CA3 subfields. Neuroscience 78:673-684, 1997.

Morioka M, Hamada J, Ushio Y, Miyamoto E. Potential role of calcineurin for brain ischemia and traumatic injury. Prog. Neurobiol. 58:1-30, 1999.

Mulkey RM, Endo S, Shenolokar S, Malenka E. Involvement of a calcineurin/inhibitor-1 phosphatase cascade in hippocampal long-term depression. Nature 369:486-488, 1994.

MülLER M. The cochlear place-frequency map of the adult and developing Mongolian gerbil. Hear. Res. 94:148-156, 1996.

Nakagawa T, Yamane H. Cytochrome $c$ redistribution in apoptosis of guinea pig vestibular hair cells. Brain Res. 847:357-359, 1999.

Nakazawa A, Usuda N, Matsui T, Hanai T, Matsushita S, Arai H, SASAKI H, Higuchi S. Localization of calcineurin in the mature and developing retina. J. Histochem. Cytochem. 49(2):187-195, 2001.

Nicotera TM, Hu BH, Henderson D. The caspase pathway in noiseinduced apoptosis of the chinchilla cochlea. J. Assoc. Res. Otolaryngol. 4(4):466-477, 2003.

ReEd JC. Cytochrome $c$ : can't live with it-can't live without it. Cell 91:559-562, 1997.

Schmiedt RA, Okamura H, Lang H, Schute BA. Ouabain application to the round window of the gerbil cochlea: a model of auditory neuropathy and apoptosis. J. Assoc. Res. Otolaryngol. 3:223-233, 2002.

SKAPER S. Poly (ADP-ribose) polymerase-1 in acute neuronal death and inflammation: a strategy for neuroprotection. Ann. N. Y. Acad. Sci. 993:217-228, 2003.

Spoendin H. Innervation patterns in the organ of Corti of the cat. Acta Otolaryngol. 67:239-254, 1969.

Spoendin H. Retrograde degeneration of the cochlear nerve. Acta Otolaryngol. 79:266-275, 1975.

Spoenduin H. Differentiation of cochlear neurons. Acta Otolaryngol. 91:451-456, 1981.

Spoendun H. Factors inducing retrograde degeneration of the cochlear nerve. Ann. Otol. Rhinol. Laryngol. Suppl. 112:76-82, 1984.

Verma IM, Stevenson JK, Schwarz EM, Antwerp DV, Miyamoto S. $\mathrm{Rel} / \mathrm{NF}-\kappa \mathrm{B} / \mathrm{I} \kappa \mathrm{B}$ family: intimate tales of association and dissociation. Genes Dev. 9:2723-2735, 1995.

Wallace RA, Tallant EA, Cheung WY. High levels of heat-labile calmodulin-binding protein (CaM-BP80) in bovine neostriatum. Biochemistry 19:1831-1837, 1980.

White JA, Burgess, Hall RD, Nadol JB. Pattern of degeneration of the spiral ganlion cell and its processes in the C57BL/6J mouse. Hear. Res. 141:12-18, 2000.

Xiao AY, Wei L, Xia S, Rothman S, Yu SP. Ionic mechanism of ouabain-induced concurrent apoptosis and necrosis in individual cultured cortical neurons. J. Neurosci. 22(4):1350-1362, 2002.

Yamasaki Y, Onodera H, Adachi K, Shozuhara H, Kogure K. Alteration in the immunoreactivity of the calcineurin subunits after ischemic hippocampal damage. Neuroscience 49(3): 545-556, 1992. 
Ylikoski J, Collan Y, Palva T. Ultrastructural features of spiral ganglion cells. Arch. Oto-Rhino-Laryngol. 104:84-88, 1978.

Yu SW, Wang H, Dawson TM, Dawson VL. Poly(ADP-ribose) polymerase-1 and apoptosis inducing factor in neurotoxicity. Neurobiol. Dis. 14(3):303-317, 2003.

Yu Z, Zhou D, Bruce-Keller AJ, Kindy MS, Mattson MP. Lack of the p50 subunit of nuclear factor- $\mathrm{kB}$ increases the vulnerability of hippocampal neurons to excitotoxic injury. J. Neurosci. 19(20):8856-8865, 1999.

Zimmerman CE, Burgess BJ, Nadol JB. Patterns of degeneration in the human cochlear nerve. Hear. Res. 90(1-2):192-201, 1995. 